\title{
REAL LINEAR CHARACTERS OF THE SYMPLECTIC MODULAR GROUP
}

IRVING REINER

1. The symplectic modular group $\Gamma_{2 n}$ consists of all integral $2 n \times 2 n$ matrices $\mathfrak{M}$ for which $\mathfrak{M} \mathfrak{F} \mathfrak{M}^{\prime}=\mathfrak{F}$, where

$$
\mathfrak{F}=\left(\begin{array}{cc}
0 & I^{(n)} \\
-I^{(n)} & 0
\end{array}\right)
$$

In order to determine all possible automorphisms of $\Gamma_{2 n}[1],{ }^{1}$ it is necessary to find all real linear characters of $\Gamma_{2 n}$, that is, all homomorphisms into $\{ \pm 1\}$. In this note we prove that $\Gamma_{2 n}$ has no nontrivial real linear characters for $n>2$, while $\Gamma_{2}$ and $\Gamma_{4}$ each have exactly one nontrivial real linear character. We shall also determine $\Gamma_{2 n}^{\prime}$, the commutator subgroup of $\Gamma_{2 n}$.

We define the symplectic direct sum $\mathfrak{M}_{1} * \mathfrak{M}_{2}$ by

$$
\mathfrak{M}_{1} * \mathfrak{M}_{2}=\left(\begin{array}{ll}
A_{1} & B_{1} \\
C_{1} & D_{1}
\end{array}\right) *\left(\begin{array}{ll}
A_{2} & B_{2} \\
C_{2} & D_{2}
\end{array}\right)=\left(\begin{array}{llll}
A_{1} & 0 & B_{1} & 0 \\
0 & A_{2} & 0 & B_{2} \\
C_{1} & 0 & D_{1} & 0 \\
0 & C_{2} & 0 & D_{2}
\end{array}\right) .
$$

Set

$$
S=\left(\begin{array}{rr}
0 & 1 \\
-1 & 0
\end{array}\right), \quad T=\left(\begin{array}{ll}
1 & 1 \\
0 & 1
\end{array}\right), \quad V=\left(\begin{array}{ll}
0 & 1 \\
1 & 0
\end{array}\right)
$$

and define ${ }^{2}$

$$
U_{0}=S+I^{(n-2)}, \quad U_{1}=V+I^{(n-2)}, \quad U_{2}=T+I^{(n-2)} .
$$

Then [2] $\Gamma_{2 n}$ is generated by $\Re_{i}=U_{i}+U_{i}^{\prime-1}(i=0,2), \mathfrak{T}_{0}=T * I^{2(n-1)}$, $\mathfrak{S}_{0}=S * I^{2(n-1)}$, and their conjugates. When $n=1$, the $\Re_{i}$ are superfluous. Next we remark that

$$
\begin{aligned}
\mathfrak{S}_{0} \mathfrak{T}_{0} & =(S T) * I=(S T)^{-2} * I, \\
\Re_{0} \Re_{2} & =U_{3}+U_{3}^{\prime}, \text { where } U_{3}=S T+I=(S T+I)^{-2}, \\
\mathfrak{T}_{0} & =\Re_{1} \Re_{2} \cdot \Re_{0} \mathfrak{T}_{0} \Re_{0}^{-1} \mathfrak{T}_{0}^{-1} \cdot\left(\Re_{1} \Re_{2}\right)^{-1} \cdot \mathfrak{S}_{0} \Re_{1} \cdot \Re_{2} \cdot\left(\mathfrak{S}_{0} \Re_{1}\right)^{-1} .
\end{aligned}
$$

Presented to the Society, December 29, 1954 under the title Characters of the symplectic modular group; received by the editors November 13, 1954 and, in revised form, November 24, 1954.

${ }^{1}$ Numbers in brackets refer to the bibliography at the end of the paper.

2 $A+B$ denotes the direct sum of the matrices $A$ and $B$. 
Therefore if $\theta$ is any real linear character of $\Gamma_{2 n}$, we must have

$$
\theta\left(\Re_{0}\right)=\theta\left(\Re_{2}\right)=\theta\left(\mathfrak{T}_{0}\right)=\theta\left(\mathfrak{S}_{0}\right)= \pm 1 .
$$

On the other hand, let $\Omega_{n}$ be the unimodular group consisting of all integral $n \times n$ matrices with determinant \pm 1 . Then for $n>2, \Omega_{n}$ is its own commutator subgroup [3]. Hence for $n>2, U_{0}$ is a product of commutators in $\Omega_{n}$, and therefore $\Re_{0}$ is in the commutator subgroup of $\Gamma_{2 n}$. Therefore $\theta\left(\Re_{0}\right)=+1$, so $\Gamma_{2 n}$ has no nontrivial real linear characters for $n>2$.

Now we must prove that there exists a homomorphism of $\Gamma_{2 n}$ into $\{ \pm 1\}$ which maps each generator $\Re_{0}, \Re_{2}, \mathfrak{T}_{0}, \mathfrak{S}_{0}$ into -1 , for the cases $n=1$ and $n=2$. This is already known for $n=1$ [3], but we give an independent proof here. Let $H$ be the normal subgroup of $\Gamma_{2 n}$ consisting of all matrices $\equiv I^{(2 n)}(\bmod 2)$. Then it is known [4] that $\Gamma_{2 n} / H \cong S_{3 n}$ for $n=1,2$, where $S_{k}$ is the symmetric group on $k$ symbols. Let $\pi$ be the homomorphism mapping $\Gamma_{2 n}$ onto $S_{3 n}$, and let $A_{3 n}$ be the alternating subgroup of $S_{3 n}$. Then $\pi^{-1}\left(A_{3 n}\right)$ is a subgroup of index 2 of $\Gamma_{2 n}, n=1,2$. Therefore $\Gamma_{2 n}$ has a nontrivial real linear character for $n=1,2$, and the previous discussion shows that it is unique, and maps each generator onto -1 .

2. Now we consider $\Gamma_{2 n}^{\prime}$, and we begin with $n=1$, the most difficult case. The commutator subgroup of $\Gamma_{2} /\{ \pm I\}$ is known [5], but we shall not use this earlier result. According to [6], $\Gamma_{2}=\{S, T\}$ has as defining relations

$$
S^{4}=T S^{-1} T S^{-1} T S=1 .
$$

Then the sum of the exponents to which $S$ (resp. $T$ ) occurs in any relation, must be of the form $4 a-b$ (resp. $3 b$ ), where $a$ and $b$ are integers. For $X \in \Gamma_{2}$ let $\alpha_{X}=$ sum of the exponents to which $S$ occurs, and let $\beta_{X}=$ sum of the exponents to which $T$ occurs, when $X$ is expressed as a power product of $S$ and $T$. Then $X \in \Gamma_{2}^{\prime}$ implies that $\alpha_{X}, \beta_{X}$ are of the form

$$
\alpha_{X}=4 a-b, \quad \beta_{X}=3 b,
$$

for integral $a, b$. On the other hand,

$$
S^{4 a-b} T^{3 b}=S^{-b} T^{3 b} \equiv\left(S^{-1} T^{3}\right)^{b}\left(\bmod \Gamma_{2}^{\prime}\right),
$$

and

$$
S^{-1} T^{3}=S^{-1} T \cdot S T^{-1} S T^{-1} S^{-1} \cdot T \in \Gamma_{2}^{\prime} .
$$

Hence $X \in \Gamma_{2}^{\prime}$ if and only if (4) holds. Consequently $T^{12} \in \Gamma_{2}^{\prime}, T^{m} \notin \Gamma_{2}^{\prime}$ for $m=1, \cdots, 11$, and we have 


$$
\Gamma_{2}=\bigcup_{m=0}^{11} T^{m} \Gamma_{2}^{\prime}
$$

Thus $^{3}\left(\Gamma_{2}: \Gamma_{2}^{\prime}\right)=12$.

Next we show how $\Gamma_{2}^{\prime}$ may be defined by means of congruences. Let

$$
H_{m}=\left\{X \in \Gamma_{2}: X \equiv I(\bmod m)\right\} .
$$

Then $H_{m}$ is a normal subgroup of $\Gamma_{2}$, and in particular $\Gamma_{2} / H_{3}$ is a group of order 24 consisting of all $2 \times 2$ matrices of determinant +1 with elements in $G F(3)$. This group contains a normal subgroup $\{C, D\}[4]$ of index 3 , where

$$
C=\left(\begin{array}{rr}
0 & 1 \\
-1 & 0
\end{array}\right), \quad D=\left(\begin{array}{ll}
1 & 1 \\
1 & 2
\end{array}\right)
$$

Hence the group $K_{3}$ consisting of all elements of $\Gamma_{2}$ congruent (mod 3$)$ to a matrix in $\{C, D\}$ is a normal subgroup of $\Gamma_{2}$ of index 3 . Therefore $\Gamma_{2}^{\prime} \subset K_{3}$.

Next we remark that $\Gamma_{2} / H_{4}$ is of order 48 , and contains the normal subgroup $\{A, E, F\}$ of order 12 generated by

$$
A=\left(\begin{array}{ll}
-1 & 1 \\
-1 & 0
\end{array}\right), \quad E=\left(\begin{array}{rr}
-1 & 0 \\
2 & -1
\end{array}\right), \quad F=\left(\begin{array}{rr}
-1 & 2 \\
0 & -1
\end{array}\right)
$$

taken mod 4. If $K_{4}$ is the set of all elements of $\Gamma_{2}$ congruent mod 4 to a matrix in $\{A, E, F\}$, then $K_{4}$ is a normal subgroup of $\Gamma_{2}$ of index 4. Therefore $\Gamma_{2}^{\prime} \subset K_{4}$. Since $K_{4} K_{3}=\Gamma_{2}$, it follows at once that

$$
\left(\Gamma_{2}: K_{3} \cap K_{4}\right)=12 \text {, }
$$

and so

$$
\Gamma_{2}^{\prime}=K_{3} \cap K_{4} \cdot{ }^{4}
$$

3. We show next that $\left(\Gamma_{4}: \Gamma_{4}^{\prime}\right)=2$, and also we determine $\Gamma_{4}^{\prime}$ by means of congruences. From [3] we find that $\Re_{0} \Re_{2}$ and $\Re_{2}^{2} \in \Gamma_{4}^{\prime}$. Hence $L=\Gamma_{4}^{\prime} \cup \Re_{2} \Gamma_{4}^{\prime}$ is a normal subgroup of $\Gamma_{4}$, and (using (3)) $\Re_{0}$, $\Re_{2}$, and $\mathfrak{T}_{0}$ are elements of $L$. Also we have

$$
\mathfrak{S}_{0} \mathfrak{T}_{0}=\mathfrak{T}_{0} \mathfrak{S}_{0} \mathfrak{T}_{0}^{-1} \mathfrak{S}_{0}^{-1}\left(\mathfrak{S}_{0} \mathfrak{T}_{0} \mathfrak{S}_{0}^{-1}\right)^{2} \mathfrak{T}_{0}^{2},
$$

so $\Im_{0} \in L$. Hence $L=\Gamma_{4}$, and therefore either $\Gamma_{4}^{\prime}=\Gamma_{4}$ or $\left(\Gamma_{4}: \Gamma_{4}^{\prime}\right)=2$.

3 This result has been obtained independently by Professor J. L. Brenner.

- The author wishes to acknowledge with thanks some helpful conversations with Professor E. V. Schenkman on the material in $\$ 2$. 
However we have already seen that $\Gamma_{4}$ contains a subgroup $K$ of index 2. Since $\Gamma_{4}^{\prime} \subset K$, we then have $\Gamma_{4}^{\prime}=K$.

Finally we remark that the previous discussion shows easily that $\Gamma_{2 n}^{\prime}=\Gamma_{2 n}$ for $n>2$.

\section{BIBLIOGRAPHY}

1. I. Reiner, Automorphisms of the symplectic modular group, Trans. Amer. Math. Soc. vol. 80 (1955) pp. 35-50.

2. L. K. Hua and I. Reiner, Trans. Amer. Math. Soc. vol. 65 (1949) pp. 415-426.

3. ——, Trans. Amer. Math. Soc. vol. 71 (1951) pp. 331-348.

4. L. E. Dickson, Linear groups, Teubner, 1901.

5. H. Frasch, Math. Ann. vol. 108 (1933) pp. 229-252, especially p. 245, footnote.

6. J. Nielsen, Danske Videnskabernes Selskab. Matematisk-Fysiske Meddelelser vol. 5, no. 18 (1924) pp. 3-29.

Institute for Advanced Study and

UNIVERSITY OF ILLINOIS 